A N N A L E S Annales de Bretagne et des Pays de l'Ouest

Anjou. Maine. Poitou-Charente. Touraine

113-3 | 2006

Prieurés et société au Moyen Âge

\title{
La naissance des prieurés de l'abbaye féminine Saint-Georges de Rennes (1024-1047)
}

Étienne Mathieu

\section{OpenEdition}

Journals

Édition électronique

URL : http://journals.openedition.org/abpo/793

DOI : 10.4000/abpo.793

ISBN : 978-2-7535-1503-1

ISSN : 2108-6443

Éditeur

Presses universitaires de Rennes

Édition imprimée

Date de publication : 30 octobre 2006

Pagination : 93-104

ISBN : 978-2-7535-0405-9

ISSN : 0399-0826

\section{Référence électronique}

Étienne Mathieu, «La naissance des prieurés de l'abbaye féminine Saint-Georges de Rennes

(1024-1047) », Annales de Bretagne et des Pays de l'Ouest [En ligne], 113-3 | 2006, mis en ligne le 30

octobre 2008, consulté le 19 avril 2019. URL : http://journals.openedition.org/abpo/793 ; DOI :

$10.4000 / a b p o .793$ 


\title{
La naissance des prieurés de l'abbaye féminine Saint-Georges de Rennes (1024-1047)
}

\author{
Étienne MATHIEU \\ Master 2 d'histoire, Université Rennes 2 Haute-Bretagne
}

Entre 1024 et 1034, Alain III, duc de Bretagne, fonde à Rennes l'abbaye Saint-Georges, une abbaye de bénédictines. Cette fondation est effectuée en présence de nombreux grands laïques et ecclésiastiques, puisque le comte de Cornouaille comme les neufs évêques de la péninsule y assistent ${ }^{1}$. Elle s'inscrit indéniablement dans le mouvement de réforme monastique qui a démarré depuis le début du $\mathrm{x}^{\mathrm{e}}$ siècle. Cette fondation prend également place dans un autre mouvement, celui de la multiplication des abbayes féminines à partir du début du $\mathrm{Xl}^{\mathrm{e}}$ siècle ${ }^{2}$. En effet, l'abbaye rennaise est en Bretagne la première abbaye féminine, si l'on fait exception du petit monastère double de Locmaria, dont l'histoire est d'ailleurs confuse pour cette période ${ }^{3}$. Avec Saint-Georges, le duc fonde une abbaye féminine autonome, c'est-à-dire un établissement qui ne dépend pas d'une autre abbaye masculine comme c'est le cas pour l'abbaye de Marcigny, qui relève du monastère de Cluny, ou encore pour l'abbaye de La Celle, liée au monastère Saint-Victor de Marseille. Cela signifie que l'abbaye possède son propre réseau prieural, un réseau dépendant uniquement de l'abbesse et de l'abbaye-mère située à Rennes ${ }^{4}$.

Le terme de prieuré, on le sait, est problématique car il recouvre une réalité aux contours mal définis, au moins pour le xle siècle. Le terme latin de prioratus ne s'impose réellement qu'à partir de la fin du XII siècle, alors qu'en parallèle l'Église s'occupe de mieux définir le fonctionnement des

1. Gullotel, Hubert, Les actes des ducs de Bretagne (944-1148), thèse de droit dactyl., Université de Paris II, 1973, $\mathrm{n}^{\circ} 28$.

2. PARISSE, Michel, "La tradition du monachisme féminin au Haut Moyen Age ", dans DALARun, Jacques (éd.), Robert d'Arbrissel et la vie religieuse dans l'Ouest de la France, Actes du colloque de Fontevraud 13-16 décembre 2001, Turnhout, 2004, p. 107-120.

3. QuAgheBeUR, Joëlle, La Cornouaille du IXe au XII siècle, Quimper, 2001, p. 204.

4. Nous nous appuyons ici sur la typologie établie par PARISSE, Michel, « Des prieurés de femmes", dans LEMAîTrRE, Jean-Loup (dir.), Prieurs et prieurés dans l'Occident médiéval, Genève, 1987, p. 115-126. 
prieurés $^{5}$. Avant cette date, il existe une multiplicité de termes désignant un prieuré (locus, obedientia, domus, monasterium, cella...) et il est difficile d'en donner une définition très précise. Joseph Avril en propose une qui semble ni trop vague, ce qui aurait pour inconvénient de voir dans le moindre bâtiment monastique un prieuré, ni trop étroite, ce qui serait problématique pour le XI ${ }^{\mathrm{e}}$ siècle et conduirait à rejeter un certain nombre de cas recevables : "Le prieuré est une dépendance qui suppose un lieu de culte, une communauté régulière, variable selon les prieurés, et un temporel à gérer ${ }^{6}$. " À l'aide de cette définition, nous avons pu repérer sept prieurés dépendant de l'abbaye de Saint-Georges. Trois sont situés dans le comté de Rennes : Saint-Georges de Grehaigne, Saint-Séglin et la Chapelle Jeanson; un dans le comté de Penthièvre : celui de Tinténiac; un dans celui de Vannes : le prieuré de l'île d'Arz; et les deux derniers dans le comté de Trégor : ceux de Pleubian et de Plougasnou. Ces sept dépendances apparaissent entre 1024/1034 et 1040/1047, période qui correspond au démarrage du grand mouvement d'efflorescence prieurale. En Bretagne, ce mouvement se caractérise par de nombreuses fondations à l'initiative des seigneurs châtelains et au profit des grandes abbayes ligériennes, notamment Marmoutier et Saint-Florent de Saumur ${ }^{7}$. Or, sur les sept fondations dépendant de l'abbaye de Saint-Georges, cinq, soit l'essentiel, sont effectuées par les ducs de Bretagne Alain III et Conan II, c'est-à-dire le fondateur de l'abbaye et son fils. Les deux dernières, la Chapelle Jeanson et SaintSéglin, sont le fait de la vicomtesse Roianteline.

Nous ne possédons pas à proprement parler d'acte de fondation et aucun acte ne mentionne la volonté explicite d'un donateur de fonder un prieuré dépendant de l'abbaye rennaise. Cependant, six donations initiales se trouvent directement à l'origine de l'apparition de ces dépendances et certains actes du cartulaire ou des pièces originales plus tardives en confirment l'existence. La naissance des prieurés de l'abbaye de Saint-Georges se démarque donc du modèle " châtelain " que nous venons d'évoquer. Nous montrerons dans un premier temps que les sept prieurés de SaintGeorges sont effectivement attestés par des actes postérieurs, puis, dans un deuxième temps, que les six donations que nous avons évoquées sont bien à l'origine de la création des sept prieurés de l'abbaye rennaise. Dans un dernier temps nous envisagerons quelles ont pu être les motifs des donations donnant lieu à l'érection de prieurés.

5. BAUTIER, Anne-Marie, « De prepositus à prior, de cella à prioratus : évolution linguistique et genèse d'une institution ", dans LEMAîTRE, Jean-Loup (dir.), Prieurs et prieurés dans l'Occident médiéval, Genève, 1987, p. 1-21.

6. Avril, Jacques, "Paroisses et dépendances monastiques au Moyen Age ", Revue Mabillon, t. 60, 1980, p. 453-517.

7. CHÉDEVILLE, André et TONNERRE, Noël-Yves, La Bretagne féodale, XI ${ }^{e}$-XIII siècle, Rennes, 1987, p. 226-227. 


\section{Les attestations tardives de l'existence des prieurés}

Comme nous l'avons dit, aucun acte du cartulaire de Saint-Georges ne mentionne la fondation d'un prieuré dépendant de cette abbaye. C'est donc par les mentions de ces prieurés dans des actes postérieurs à leur fondation que nous connaissons leur existence. Le premier prieuré de l'abbaye apparaissant dans un acte est celui de Saint-Georges de Grehaigne. Entre 1047 et 1057, Rogerius, un fidèle du comte Eudes, l'oncle de Conan II, concède toute la terre qu'il possède dans la paroisse de Grehaigne au monasterium de Grehaigne ${ }^{8}$. Le terme de monasterium désigne ici le prieuré dépendant de Saint-Georges. Vers 1060, l'abbesse de Saint-Georges, Adèle, passe un accord avec un certain Gauthier, pour que celui-ci soit le prévôt de l'abbaye dans la paroisse de Pleubian. Il devra écouter les conseils des moniales résidant dans l'obedientia de ce lieu : ce terme atteste l'existence d'un prieuré dans la paroisse de Pleubian peu après le milieu du XI ${ }^{\mathrm{e}}$ siècle $^{9}$.

On remarquera que pour ces deux premières mentions de prieurés, les termes employés par les actes du $\mathrm{XI}^{\mathrm{e}}$ siècle sont variables et que prioratus n'est pas usité. En revanche, nous ne rencontrons les premières mentions confirmant l'existence des autres prieurés de Saint-Georges que dans des actes du XIII ${ }^{\mathrm{e}}$ siècle, dans lesquels ils sont toujours désignés par le terme de prioratus. Celui de Tinténiac apparaît dans un acte daté de 1224 relatant un accord entre l'abbesse de Saint-Georges et le prêtre de Tinténiac ${ }^{10}$. Celui de Saint-Séglin est mentionné dans une lettre datant de 1299 écrite par Alain de Châteaugiron ${ }^{11}$. Le prieuré de l'île d'Arz n'est pas directement désigné, mais un acte de 1251 évoque la prieure de l'île d'Arz, ce qui nous permet d'en déduire l'existence d'un prieuré ${ }^{12}$. Notons également que nous retrouvons le prieuré de Saint-Georges de Grehaigne désigné par le terme de prioratus dans un acte daté de $1233^{13}$. Le prieuré de la Chapelle Jeanson est mentionné dans un acte du XIII ${ }^{\mathrm{e}}$ siècle relatant un partage de dîme ${ }^{14}$. Quant au prieuré de Plougasnou, nous n'en avons aucune trace, même dans les actes du XIII ${ }^{\mathrm{e}}$ siècle. En revanche, il est qualifié de prieuré dans les aveux rendus au Roy datant de $1664^{15}$. L'existence des sept prieurés est donc bien confirmée par des actes postérieurs à leur fondation. En outre, dans deux

8. Cartulaire de l'abbaye de Saint-Georges de Rennes, éd. par La Bigne Villentuve, P., Rennes, $1876, \mathrm{n}^{\circ} 29$.

9. Ibidem, $\mathrm{n}^{\circ} 19$.

10. Ibid., $\mathrm{n}^{\circ} 24$ de l'appendice.

11. Ibid., $\mathrm{n}^{\circ} 52$ de l'appendice.

12. Ibid. $\mathrm{n}^{\circ} 32$ de l'appendice.

13. Ibid., $\mathrm{n}^{\circ} 29$ de l'appendice.

14. Nous ne connaissons cet acte que par une mention de Guillotin de Corson. Celui-ci renvoie aux archives d'Ille-et-Vilaine mais nous ne l'y avons pas retrouvé. Aussi pouvonsnous nous demander si le prieuré est réellement mentionné par le terme de prioratus. Voir Gulllotin De CoRson, abbé, Pouillé historique de l'archevêché de Rennes, Paris, 1883, p. 279 .

15. Cartulaire de Saint-Georges..., op. cit., p. 345-385. 
cas, ces attestations interviennent quelques années seulement après ce que nous estimons être les donations donnant naissance aux prieurés considérés. Ce sont de celles-ci dont il va être à présent question.

\section{Les donations originelles et l'origine des prieurés}

Ces sept donations possèdent des caractéristiques communes. La première est l'importance des biens concédés, qui laisse penser qu'elles ont pu donner lieu à l'érection d'un prieuré. En outre, à une exception près, elles sont les premières donations faites en faveur de l'abbaye dans les paroisses concernées.

La donation grâce à laquelle sera fondé le prieuré de Tinténiac est rapportée par l'acte de fondation de l'abbaye Saint-Georges elle-même. Comme le pense Hubert Guillotel, il est probable que cet acte a été rédigé après la fondation effective, une fois que celle-ci eut prouvé sa viabilité. Le texte de l'acte aurait alors pris en compte quelques donations postérieures, comme celle de Tinténiac dont la datation peut être située vers $1024-1034^{16}$. Alain III concède aux moniales le vicus de Tinténiac, avec son église. Ce vicus est qualifié de "non exiguum ", ce qui laisse entrevoir l'importance de la donation. Le duc exempte en outre l'abbaye de toute taxe publique. Le terme d'immunité n'apparaît pas, mais la concession y ressemble fortement. D'ailleurs il apparaît dans un autre acte d'Alain III : celui rapportant la donation du domaine de Livré à Saint-Florent de Saumur ${ }^{17}$. Deux actes postérieurs du cartulaire de Saint-Georges permettent de se rendre réellement compte de l'étendue de la concession, que le simple terme de vicus ne permet pas d'appréhender. Le premier est un accord, datant d'environ 1060, passé entre l'abbesse de Saint-Georges, Adèle, et le chapelain du seigneur de Tinténiac, Guillaume l'Ismaëlite ${ }^{18}$. Cet accord porte sur la répartition des droits paroissiaux entre l'abbaye et le chapelain. L'abbesse et le chapelain auront chacun la moitié des offrandes des fidèles vivant dans le castellum de Tinténiac; en revanche, Saint-Georges percevra la totalité des offrandes des fidèles habitant dans le reste de la paroisse. Donc, lorsque le duc a donné le vicus de Tinténiac à l'abbaye rennaise, il lui a dans le même temps concédé la paroisse et l'ensemble des droits paroissiaux. Or dans la première moitié $\mathrm{du} \mathrm{XI}{ }^{\mathrm{e}}$ siècle la paroisse de Tinténiac est une paroisse de grandes dimensions, d'environ 7500 hectares, qui sera divisée au XIII ${ }^{\mathrm{e}}$ siècle en neuf paroisses ${ }^{19}$. Un second acte, datant lui aussi de 1060 , nous permet d'appréhender les droits fonciers et banaux concédés à l'abbaye en même temps que le vicus. Il s'agit d'un censier dans lequel sont recensées les villae possédées par les seigneurs de Tinténiac au titre du

16. Gullotel, Hubert, Les actes des ducs..., op. cit., $\mathrm{n}^{\circ} 28$.

17. Ibidem, $\mathrm{n}^{\circ} 13$.

18. Cartulaire de Saint-Georges..., op. cit., $\mathrm{n}^{\circ} 3$.

19. Le Boulanger, Françoise, L'évolution du peuplement dans la région de Tinténiac au Moyen Âge. Archéologie du parcellaire - activités humaines, mémoire de maîtrise, dactyl., Université de Rennes 2, 1988, p. 86-88. 
casamento de Saint-Georges ${ }^{20}$. On constate que les droits perçus par les seigneurs de Tinténiac le sont sur l'ensemble du territoire paroissial de Tinténiac. Sachant que la seigneurie de Tinténiac s'est établie après la donation initiale à Saint-Georges et que, comme le stipule le censier, les seigneurs de Tinténiac tiennent leurs droits de l'abbaye de Saint-Georges, nous pouvons supposer que l'abbaye les a reçus par la donation d'Alain III ${ }^{21}$. La donation initiale de Tinténiac est donc composée d'un vicus, de son église et de l'ensemble du territoire paroissial de Tinténiac, avec les droits afférents à cette possession, qu'ils soient banaux, fonciers ou ecclésiastiques. Il s'agit donc d'une concession considérable.

Les donations initiales de Pleubian et de Plougasnou se ressemblent. Il existe deux versions de la donation initiale de Plougasnou, l'une étant plus détaillée que l'autre ${ }^{22}$. La plus longue des deux a d'ailleurs probablement été rédigée à l'aide de l'acte relatant la donation de Pleubian. Nous retrouvons en effet une énumération de droits étrangement semblable dans les deux actes ${ }^{23}$. La donation de Pleubian est faite par le duc Alain III entre 1034 et 1040. Il concède aux moniales " parrochiam in Britannia que vocatur Plubihan cum omnibus consuetudinibus nobis inde pertinentibus, cum equitibus et villanis, cum terra culta et inculta, cum carrucis etiam bobus, omnique dominio in perpetuum possidendam ${ }^{24}$ ". Selon Michel Brand'Honneur, le terme d'equites renverrait aux droits et services possédés sur les chevaliers, pour que l'abbaye de Saint-Georges puisse participer à l'ost comtal. Il s'agirait par conséquent de chevaliers chasés sur une terre et qui doivent le service militaire ${ }^{25}$. L'énumération des droits donnés à l'abbaye révèle en tout cas que la paroisse concédée est aussi une seigneurie ${ }^{26}$. Quant à l'église paroissiale, les moniales l'ont probablement reçue dans le même temps, deux bulles papales de 1164 et 1208 venant confirmer la possession de cette église par l'abbaye ${ }^{27}$. Ainsi, Alain III a concédé aux moniales une paroisse, qui se révèle être également une seigneurie, avec en outre l'église paroissiale. La donation de Plougasnou est effectuée en 1040 par Berthe et le jeune duc Conan II, au moment de la mort d'Alain III, leur époux et père. La première version de la donation attribue aux moniales la paroisse en intégralité, avec les terres cultivées et incultes, les champs et les forêts et

20. Cartulaire de Saint-Georges..., op. cit., $\mathrm{n}^{\circ} 2$.

21. Au sujet de la naissance de la seigneurie de Tinténiac, voir BRAND'HONNEUR, Michel, Manoirs et châteaux dans le comté de Rennes. Habitat à motte et société chevaleresque (XI XII siècle), Rennes, 2001, p. 105-108.

22. Cartulaire de Saint-Georges..., op. cit., $\mathrm{n}^{\circ} 18$ et $\mathrm{n}^{\circ} 27$.

23. Ibidem, $\mathrm{n}^{\circ} 15$ et $\mathrm{n}^{\circ} 27$.

24. Ibid., $\mathrm{n}^{\circ} 15$ : "Une paroisse de Bretagne qui s'appelle Pleubian avec toutes les coutumes que nous tenions en ce lieu, avec les chevaliers et les vilains, avec la terre cultivée et inculte, avec les charrues et les bœufs, et tout le pouvoir en possession perpétuelle."

25. Brand'HONNEUR, Michel, Manoirs et châteaux..., op. cit., p. 149.

26. Ріснот, Daniel, Le village éclaté. Le paysage rural dans l'Ouest de la France, Rennes, 2001, p. 305.

27. Cartulaire de Saint-Georges..., op. cit., p. 168-176. 
les rivières coulant vers la mer ${ }^{28}$. La seconde explicite un certain nombre de droits, notamment sur les equites, comme dans le cas de Pleubian. Berthe et Conan II ont-ils réellement concédé ces droits lors de la donation, la seconde version ne faisant alors que les détailler pour affermir les droits de Saint-Georges, ou cette seconde version est elle un faux? La question reste en suspens. En tout cas, il nous semble que le cas de Plougasnou est semblable à celui de Pleubian : une paroisse, qui est à la fois une seigneurie, avec son église paroissiale, dont la possession est elle aussi confirmée par les bulles pontificales.

L'acte de donation de l'île d'Arz est extrêmement court et relate laconiquement qu'entre 1024 et 1034 Alain III concède à l'abbaye qu'il a fondée la moitié de l'île avec la moitié des droits et coutumes qu'il y possédait ${ }^{29}$. En outre, les bulles pontificales de 1164 et 1208 montrent que les moniales y possédaient également, à cette époque, la moitié des droits paroissiaux. Ces droits faisaient certainement partie de la concession effectuée par Alain III. Saint-Georges partageait aussi probablement l'église paroissiale avec l'abbaye de Saint-Gildas-de-Ruys, qui possédait l'autre moitié de l'île ${ }^{30}$. Cependant, comme dans les cas de Pleubian et Plougasnou, la concession de l'église n'est pas clairement explicitée.

L'acte de donation initiale de Saint-Georges de Grehaigne ne mentionne pas le nom du donateur. Cependant, les bulles pontificales déjà évoquées nous permettent d'y voir l'œuvre de Conan II, duc de Bretagne, fils du fondateur de l'abbaye, Alain III. Entre 1040 et 1047, Conan II concède la villa de Grehaigne, avec l'église qui s'y trouve, la moitié du marais adjacent à la villa et la moitié du droit de pêche ${ }^{31}$. Selon Daniel Pichot, cette villa peut en fait être assimilée à un village ${ }^{32}$. D'ailleurs les bulles pontificales de 1164 et 1208 renforcent cette impression. Elles évoquent les différents droits concédés par Conan II au moment de cette donation : "Ecclesiam Sancti Georgii cum hominibus ejusdem loci et aliis pertinenciis suis; homines in cimiterio Sancti Georgii commorantes, ad ejusdem monasterii proprietatem per-

28. Ibidem, $\mathrm{n}^{\circ} 18$.

29. Ibid., $\mathrm{n}^{\circ} 14$.

30. Ibid., $\mathrm{n}^{\circ} 32$ de l'appendice.

31. Ibid., $\mathrm{n}^{\circ}$ 26. La datation de ce document nécessite une explication. Hubert Guillotel, au regard de l'absence de témoins, date l'acte de manière large, entre 1040 et 1066 . Cependant, une donation est effectuée par un certain Rogerius, fidèle d'Eudes, l'oncle de Conan II, en faveur du monasterium de Grehaigne, entre 1040 et 1057. Nous proposons cette fourchette car elle correspond à la période de " régence " d'Eudes (jusqu'en 1047), puis d'affrontement entre l'oncle et le neveu. Après cette période, Eudes se replie en Domnonée et une intervention de sa part si loin de son apanage paraît plus qu'improbable. Le terme monasterium indique l'existence d'un prieuré à Grehaigne, or nous estimons que seule la donation de Conan II a pu susciter l'érection d'un prieuré par l'abbaye de Saint-Georges à Grehaigne. En outre, l'abbaye de Saint-Georges, à travers son abbesse, ayant pris parti pour Eudes durant le conflit, il paraît plausible de restreindre la fourchette chronologique à 1040-1047, c'est-à-dire avant le début du conflit, voir GuILLOTEL, Hubert, Les actes des ducs..., op. cit., n ${ }^{\circ} 58$.

32. Ріснот, Daniel, Le village éclaté..., op. cit., p. 163. 
tinentes, ex bone memorie comitis Conano quicquid juris habebat in predicta villa de Grihannia, tam in hominibus quam aliis possessionibus ${ }^{33}$. "L'accent est ici mis sur les hommes. La villa est dotée d'un cimetière habité, ce qui est courant en Bretagne et souvent à l'origine du développement d'un village $^{34}$. La donation de Grehaigne est donc composée d'un village et de son église, et il est même possible que la villa recouvre le territoire paroissial. Un dernier détail est à relever : l'abbaye de Saint-Georges avait reçu un bien à proximité de Grehaigne avant la donation de Conan II, ce qui n'était pas le cas dans les autres donations. Entre 1024 et 1034, la comtesse Havoise, mère d'Alain III, avait en effet concédé aux moniales une portion de terre située au voisinage de Grehaigne ${ }^{35}$. Cependant, il ne s'agissait pas de l'élément principal de la donation, ce qui laisse présager la modestie du bien concédé. Elle n'a pas suscité l'érection d'un prieuré, ce que la donation de Conan II, en revanche, a produit.

Les donations concernant Saint-Séglin et la Chapelle Jeanson sont effectuées de manière simultanée par la vicomtesse Roianteline, l'épouse du vicomte d'Alet Hamon I ${ }^{\mathrm{er}}$, entre 1024 et 1034. Cette dernière concède alors aux moniales « capellam Jeanson cum omnibus appendiciis [...] Ecclesiam Sancti Sisginnuni cum una medietaria dum vixerit, et post mortem suam omnem terram ipsius plebis que sibi competit ${ }^{36}$ ". La Chapelle-Jeanson est probablement une église paroissiale. Un censier $\mathrm{du} \mathrm{XII}^{\mathrm{e}}$ siècle fait apparaître un territoire divisé en une trentaine de tenures, ce qui est déjà très conséquent ${ }^{37}$. Il est donc probable que l'expression cum appendiciis recouvre dès l'origine un domaine très étendu. À Saint-Séglin, la vicomtesse concède l'église paroissiale et une métairie, soit, à l'époque, une partie de la réserve seigneuriale. Une clause prévoit qu'après la mort de Roianteline les moniales recevront le reste des possessions de la vicomtesse dans cette paroisse. Dans les deux cas la donation se compose donc d'une église et d'un ensemble foncier.

Ces deux éléments sont présents dans les sept donations initiales et constituent le cœur des établissements prieuraux. Par ailleurs, il s'agit à chaque fois, sauf à Grehaigne, des premières donations effectuées dans les lieux concernés, et même des seules donations d'une telle importance dans ces paroisses jusqu'à la fin du XIII ${ }^{\mathrm{e}}$ siècle. Enfin, tous ces lieux sont situés à une certaine distance de Rennes et donc de l'abbaye-mère. Ces différents éléments permettent de penser que ces donations se trouvent bien direc-

33. Cartulaire de Saint-Georges..., op. cit., p. 173 : « L'église Saint-Georges de Grehaigne, avec les gens du lieu et tout ce qui leur appartient, les hommes séjournant dans le cimetière, sont la propriété du même monastère, par un don du comte Conan de bonne mémoire, qui avait tout ce droit dans la susdite villa de Grehaigne, tant sur les hommes que sur les possessions."

34. Ріснот, Daniel, Le village éclaté..., op. cit., p. 128-135.

35. Cartulaire de Saint-Georges..., op. cit., $\mathrm{n}^{\circ} 10$.

36. Ibidem, $\mathrm{n}^{\circ} 12$ : " La Chapelle Jeanson avec tout ce qui lui est attenant [...]. L'église Saint-Séglin avec une métairie tant qu'elle vivra et, après sa mort, toute la terre de cette même paroisse qui lui appartient."

37. ChÉdeville, André et Tonnerre, Noël-Yves, La Bretagne féodale..., op. cit., p. 213. 
tement à l'origine de l'érection de prieurés. En outre, comme le montrent les cas de Pleubian et Grehaigne, dont les prieurés sont attestés peu après la donation initiale, il est probable que les donations et les constructions de prieurés soient concomitantes.

A contrario, tous ces éléments nous amènent à rejeter trois cas litigieux. À côté des sept prieurés clairement identifiés il existe en effet trois cas problématiques. Le premier concerne le domaine d'Acigné, situé à l'est de Rennes. Selon André Dufief, l'abbaye de Saint-Georges possède un prieuré dans cette localité ${ }^{38}$. Mais aucun acte ne fait référence à celui-ci, pas même les aveux rendus au Roy qui évoquent, non un prieuré, mais un manoir dans la paroisse d'Acigné ${ }^{39}$. En outre, les moniales ne possèdent pas l'église paroissiale, ce qui est pourtant une caractéristique commune aux sept prieurés cités plus haut. Enfin, il nous semble que la faible distance séparant l'abbaye-mère de la paroisse d'Acigné est un élément supplémentaire allant à l'encontre de l'existence d'un prieuré dans cette paroisse. Le deuxième cas litigieux concerne la localité de Chavagne. Entre 1024 et 1034, la vicomtesse Roianteline concède à l'abbaye de Saint-Georges une communauté de neuf sœurs qu'elle a fondée dans la paroisse de Chavagne ${ }^{40}$. Elle souhaite que cette communauté intègre le monastère rennais en raison de ses difficultés à survivre, neuf sœurs étant en effet un effectif assez important. Ce petit monastère aurait pu devenir un prieuré dépendant de l'abbaye de Saint-Georges, mais plusieurs indices laissent penser que ce n'est pas le cas. Premièrement, il disparaît complètement des actes de l'abbaye par la suite. Deuxièmement, il est probable que les biens attachés à ce monastère étaient insuffisants pour assurer la survie de la communauté. Troisièmement, comme dans le cas d'Acigné, les moniales ne possèdent pas l'église paroissiale de Chavagne qu'elles ont du rétrocéder au duc Alain III entre 1034 et $1040^{41}$. Dernièrement, la proximité entre Chavagne et Rennes ne plaide pas en faveur de l'existence d'un prieuré. Le dernier cas litigieux se situe dans le bourg de Saulnières. À la fin du XI ${ }^{\mathrm{e}}$ siècle, Giron fils d'Ansquetil concède aux moniales une domus dans le bourg de Saulnières ${ }^{42}$. Cependant, ce don ne s'accompagne pas de concessions de terres, ni de l'église paroissiale. Le temporel semble donc trop modeste pour que les moniales y établissent un prieuré.

Les six donations évoquées, effectuées par Alain III, Conan II et la vicomtesse Roianteline, sont donc à l'origine des sept prieurés dépendant de l'abbaye de Saint-Georges. Aucun des actes ne mentionne une quelconque volonté de fonder un prieuré de la part de ces trois donateurs. Mais les actes donnent tout de même quelques motivations claires et les terres concédées permettent d'en proposer d'autres, moins explicites.

38. DuFIEF, André, Les cisterciens en Bretagne, XII -XIII siècles, Rennes, 1997, p. 230.

39. Cartulaire de Saint-Georges..., op. cit., p. 345-385.

40. Ibidem, $\mathrm{n}^{\circ} 12$.

41. Ibid., $n^{\circ} 9$.

42. Ibid., $\mathrm{n}^{\circ} 39$ 


\section{Les motifs des donations}

La première caractéristique commune à l'ensemble des donations est que les donateurs n'expriment pas ouvertement le souhait de fonder un prieuré. Les seules raisons explicitées dans les actes de donation sont religieuses. Toutes ces donations sont en effet effectuées pro anima, donc pour le salut du donateur et celui de sa famille qui se trouve associée en permanence à la donation. La donation est indéniablement un geste rédempteur, grâce auquel le donateur entend bénéficier de l'intercession des religieuses qui prient pour son âme ${ }^{43}$. La donation de Plougasnou est ainsi faite à la mort d'Alain III, par sa femme et son fils, pour le salut du défunt. Cette motivation explicite est présente dans l'ensemble des exemples dont nous disposons, ce qui n'est guère surprenant.

Comme nous l'avons signalé, le " modèle châtelain " de fondation des prieurés, que l'on pourrait caractériser par la création d'un prieuré à proximité du château dans le but de légitimer et de sacraliser le pouvoir du résident, n'est guère pertinent pour les sept cas qui nous préoccupent. En effet, cinq des prieurés sont fondés sur des biens concédés par les ducs de Bretagne, dont le siège du pouvoir est à Rennes, cité où a été fondée l'abbaye qui, elle, peut, pour partie, répondre à ces critères. Quant aux deux autres prieurés, les terres sur lesquelles ils sont fondés n'entrent pas non plus dans cette catégorie. En effet, les concessions faites par la vicomtesse Roianteline sont liées à la petite communauté de Chavagne. Les biens donnés par la vicomtesse doivent servir à l'entretien des nouvelles moniales et l'acte n'évoque en aucun cas la fondation d'un prieuré sur les terres concédées par Roianteline. En outre, les donations de La Chapelle-Jeanson, située dans l'évêché de Rennes à proximité de Fougères, et de Saint-Séglin, située dans le sud de l'évêché d'Alet, ressemblent à un abandon de terres que leur éloignement du cœur des possessions familiales rend difficile à gérer. En effet, il y a à cette époque un glissement progressif des possessions familiales des vicomtes d'Alet de l'évêché de Rennes vers celui de Dol et le nord de celui d'Alet ${ }^{44}$. Les terres concédées par la vicomtesse proviennent d'ailleurs peut-être de son douaire ou de sa dot, soit des biens qui ne concernent pas le cœur des possessions de la famille vicomtale ${ }^{45}$. Pour la même raison, Conan II doterait le prieuré Saint-Sauveur-des-Landes de droits qu'il serait sur le point de perdre en raison du recul du pouvoir comtal face à la seigneurie de Fougères ${ }^{46}$.

43. MAZEL, Florian, La noblesse et l'Eglise en Provence, fin $X^{e}$-début XIV : l'exemple des familles d'Agoult-Simiane, de Baux et de Marseille, Paris, 2002, p. 138-145.

44. Guillotel, Hubert, "Des vicomtes d'Alet aux vicomtes de Poudouvre ", Annales de la société d'Histoire et d'Archéologie de l'arrondissement de Saint Malo, 1990, p. 201-215.

45. LEMESLE, Bruno, La société aristocratique dans le Haut-Maine (XI et XII siècle), Rennes, 1999, p. 124-134.

46. HÉNOT, Aurélien, Les moines, le châtelain et les hommes. Le rôle politique et social des prieurés de Marmoutier près de Gahard et dans les seigneuries banales de Fougères et de Vitré (XI'-XIII siècle), mémoire de maîtrise dactyl., Université de Rennes 2, 1998, p. 36-37. 
Sur les cinq donations ducales, deux peuvent répondre à des motifs plus "politiques", dans le sens où l'abbaye semble utilisée comme un agent dans le cadre des projets ducaux de contrôle territorial, à l'intérieur du comté de Rennes d'une part, face aux ducs de Normandie d'autre part ${ }^{47}$. Tinténiac est un espace habité puisque s'y trouve un vicus qualifié de non exiguum. Le terme de vicus indique souvent une agglomération remontant à la haute antiquité, tandis que le suffixe -ac attesterait une origine galloromaine : tout cela prouve l'ancienneté de la présence humaine ${ }^{48}$. La région est aussi traversée par deux anciennes voies romaines, ce qui en fait un point de passage important ${ }^{49}$. Cependant, l'espace local semble également à mettre en valeur : la répartition des toponymes laisse apparaître une région boisée dans la première moitié du XI ${ }^{\mathrm{e}}$ siècle ${ }^{50}$. La zone de Tinténiac est donc stratégique. En la confiant aux moniales de Saint-Georges, il nous semble que le duc Alain III essaie de la soustraire aux ambitions des seigneuries qui environnent la région et commencent à se développer. En employant une expression qui s'applique aux forêts, nous pourrions parler de " mise en défens " de la région de Tinténiac.

Entre 1024 et 1030, les ducs de Normandie, Richard II puis Robert le Magnifique s'emparent d'une partie du comté de Rennes située au nordest, entre la Sélune, le Couesnon et le Tronçon. En 1035, Robert décède laissant un héritier mineur, Guillaume le Bâtard, futur Conquérant. Alain III ayant la garde de celui-ci, le conflit avec la Normandie est apaisé. En revanche, en 1040, Conan II succède à son père et Guillaume échappe à la tutelle bretonne. La question normande revient sur le devant de la scène. Conan II opèrerait alors, durant les années 1040-1047, une restructuration du secteur nord-est du comté de Rennes pour stabiliser la frontière, en renforçant la seigneurie de Fougères. Il crée un véritable pôle de pouvoir qui étend ses ramifications jusqu'à Pontorson et le confie à Main, ancien titulaire de la villicatio de Louvigné qui se trouve être un fidèle du jeune duc ${ }^{51}$. La concession de la villa de Grehaigne à l'abbaye de Saint-Georges, entre 1040 et 1047, semble s'intégrer à cette réorganisation du secteur nordest du Rennais. Grehaigne se situe sur la frontière avec la Normandie, juste en face de Pontorson, qui est un point de passage obligé pour entrer dans la péninsule ${ }^{52}$. Mais pourquoi la confier aux moniales? Peut-être en raison du caractère d'asile des terres monastiques, qui peut avoir un effet dis-

47. Cette « utilisation " de l'abbaye au profit de la politique ducale renvoie à une conception néo-carolingienne du pouvoir qui prévaut sous Alain III et Conan II, même si le règne de ce dernier est en partie un moment de transition vers une conception influencée par la réforme grégorienne. Voir MATHIEU, Étienne, L'aristocratie et l'Église en Bretagne, fin $X^{e}$ début XII siècle : l'exemple des familles ducales et de Dol-Combourg, mémoire de master 2 dactyl., Université de Rennes 2, 2005, p. 23 -63.

48. ChÉDEVILle, André et TonnerRe, Noël-Yves, La Bretagne féodale..., op. cit., p. 396.

49. Le Boulanger, Françoise, L'évolution du peuplement..., op. cit., p. 137-150.

50. Ibidem, p. 25-62.

51. BRAND'HONNEUR, Michel, Manoirs et châteaux..., op. cit., p. 111-113.

52. ChÉdeville, André et Tonnerre, Noël-Yves, La Bretagne féodale..., op. cit., p. 374. 
suasif sur un éventuel agresseur. Par ailleurs, les bourgs monastiques peuvent tout à fait assumer localement une fonction de défense ${ }^{53}$.

Les trois dernières donations effectuées par les ducs concernent des terres éloignées du comté de Rennes. Les ducs donnent ainsi à l'abbaye rennaise une assise territoriale qui correspond, globalement, à celle sur laquelle s'exerce réellement leur pouvoir. Il est difficile d'éclairer les raisons de ces donations. Deux points peuvent cependant être soulignés. En premier lieu, les paroisses de Pleubian et de Plougasnou sont situées dans un véritable désert monastique qui va de Saint-Jacut à Saint-Mathieu, alors que par ailleurs la côte nord de la Bretagne n'est pas inoccupée ${ }^{54}$. Il y a peut-être derrière ces donations la volonté ducale de densifier le réseau monastique de cette zone, une volonté qui reflèterait bien la conception néo-carolingienne du pouvoir ducal. En deuxième lieu, la situation frontalière de la paroisse de Plougasnou avec le Léon peut renvoyer à des motifs proches de ceux de la donation de Grehaigne, puisqu'à ce moment les vicomtes de Léon semblent être plutôt remuants ${ }^{55}$. Mais il ne s'agit là que d'hypothèses fragiles.

Les donations initiales sur lesquelles vont être érigés les sept prieurés de l'abbaye de Saint-Georges sont effectuées entre 1024 et 1047. Il s'agit des seules donations qui, par leur importance, peuvent donner naissance à un prieuré. Dans ces domaines, l'abbaye possède un patrimoine important et l'église paroissiale, qui sert également d'église prieurale. En outre, ces domaines sont situés à une distance relative de l'abbaye-mère. Les actes ne mentionnent pas la fondation des prieurés stricto sensu, mais il est probable qu'elle soit concomitante de la donation, comme dans les cas de Pleubian ou Grehaigne. Les donateurs n'expriment pas le souhait de fonder des prieurés sur les terres qu'ils concèdent à Saint-Georges. En conséquence, il est probable que ces érections résultent du besoin de gérer les biens concédés et soient donc d'abord le fruit d'une volonté abbatiale, même si l'on ne peut ignorer le lien très fort qui unit l'abbaye aux ducs de Bretagne entre 1024/1034 et 1047.

53. CHÉDEVILLE, André, «La guerre des bourgs », dans MoRnEt, Elisabeth (dir.), Campagnes Médiévales : l'homme et son espace. Etudes offertes à Robert. Fossier, Paris, 1995, p. 501-512. 54. ChÉdeville, André et Tonnerre, Noël-Yves, La Bretagne féodale..., op. cit., p. 229.

55. Guillotel, Hubert, "Les vicomtes de Léon au XI ${ }^{\mathrm{e}}$ et XII ${ }^{\mathrm{e}}$ siècle ", Mémoires de la Société d'Histoire et d'Archéologie de Bretagne, t. 51, 1971, p. 29-51. 


\section{RÉSUMÉ}

Cet article s'intéresse à la naissance des sept prieurés de l'abbaye de SaintGeorges, abbaye féminine située à Rennes. Partant du constat que ces sept prieurés n'ont donné lieu à aucun acte de fondation, il montre qu'ils ont été érigés sur des biens concédés entre 1024 et 1047 par les ducs de Bretagne, Alain III et Conan II, pour cinq d'entre eux, et par la vicomtesse Roianteline pour les deux derniers. Puis il s'attache aux motivations de ces trois donateurs pour résoudre la contradiction entre l'absence d'acte de fondation d'une part, l'érection de prieurés d'autre part.

\section{ABSTRACT}

The subject of this paper is the seven priories of the abbey of Saint-Georges in Rennes. The author, although it doesn't exist any act of foundation, demonstrates that these priories have been erected on land conceded by the dukes of Brittany, Alain III and Conan II, and the viscount Roianteline between 1024 and 1047. Then he examines the motivations of these three donators to explain the birth of the priories. 